\title{
Exploring preconception health and nutrition beliefs amongst adults of childbearing age in Northern Ireland: a qualitative analysis
}

\author{
L. McGowan ${ }^{1,2}$, E. Lennon-Caughey ${ }^{2}$, C. Chun ${ }^{3}$, M.C. McKinley ${ }^{1,2}$ and J.V. Woodside ${ }^{1,2}$ \\ ${ }^{1}$ Centre for Public Health, School of Medicine, Dentistry and Biomedical Science, Queen's University Belfast, \\ Belfast, $U K$, \\ ${ }^{2}$ Institute for Global Food Security, School of Biological Sciences, Queen's University Belfast, Belfast, UK and \\ ${ }^{3}$ National University of Ireland, Galway, Ireland
}

'Preconception health' $(\mathrm{PCH})$ is a term used to describe the health status of males and females prior to pregnancy. There is compelling evidence for the role of $\mathrm{PCH}$ behaviours such as diet, smoking and alcohol consumption in determining successful pregnancy outcomes and regarding the health of mother and baby ${ }^{(1)}$. PCH strategies aim to optimise the health of future offspring via improved parental health for planned/unplanned pregnancies. Greater emphasis is being placed upon PCH amongst research and public health, yet there is limited evidence on PCH from the perspective of adults in Northern Ireland (NI). Objective: This research qualitatively explored beliefs, knowledge and attitudes on PCH amongst adults of childbearing age in NI.

A descriptive qualitative focus group study was undertaken with healthy males and females (18-45 years) October 2018-July 2019. Five groups were held ( $\mathrm{n}=2$ rural; $\mathrm{n}=3$ urban), with a range of males and females, with and without children. A semi-structured topic guide was devised based on previous literature ${ }^{(2)}$. All groups were conducted with two researchers trained in qualitative research methods. Focus groups explored understanding/prior knowledge of $\mathrm{PCH}$, beliefs and attitudes towards $\mathrm{PCH}$ support, and personal views on what's important for PCH. Focus groups were transcribed verbatim and analysed using thematic analysis ${ }^{(3)}$. Full ethical approval was granted from Queen's University Belfast, School of Biological Sciences Research Ethics Committee.

Twenty-one males and females of childbearing age participated in the research; with young single females representing the largest demographic (62\% female; 52\% aged 18-25 years). Discussions revealed a lack of awareness of the importance of PCH and a sense of reluctance to visit a doctor regarding $\mathrm{PCH}$, favouring online/internet approaches. Five themes identified included: PCH education, $\mathrm{PCH}$ awareness, wider knowledge networks/support, optimal parental health (including diet/nutrition beliefs, often inaccurate), and attitudes/emotions towards PCH. The role of males in PCH was not well understood e.g., 'the woman has to do all that. ..[PCH]'.

This study highlighted a lack of detailed awareness surrounding the importance of $\mathrm{PCH}$ per se, despite general agreement that health and nutrition status should be optimal at this time. It identified a willingness to learn more about PCH, creating an opportunity to improve $\mathrm{PCH}$ awareness via evidence-based education, social media campaigns, and within healthcare systems in a life-course approach.

\section{Acknowledgements}

Thanks to all participants involved in this study for taking the time to share their personal views.

\section{References}

1. Stephenson J, Heslehurst N, Hall J, et al. (2018) The Lancet 391, 1830-1841.

2. Bortolus R, Oprandi N \& Morassutti F (2017) BMC Pregnancy Childbirth 17.

3. Braun V \& Clarke V (2006) Qual Res Psychol 3, 77-101 